\title{
Numerical study on onset of oscillatory thermocapillary flow in rectangular liquid pool
}

\author{
HU WenRui ${ }^{*}$, TANG ZeMei \& LI Kai \\ Key Laboratory of Microgravity (National Microgravity Laboratory), Institute of Mechanics, Chinese Academy of Sciences, \\ Beijing10090, China
}

Received July 21, 2009; accepted October 19, 2009; published online March 20, 2010

\begin{abstract}
Thermocapillary flow in a rectangular liquid pool of large Prandtl fluid $(\operatorname{Pr}=105.6)$ is numerically studied in microgravity. Oscillatory thermocapillary flow arises when the imposed temperature difference between the sidewalls exceeds a critical value. The fluctuations of the oscillatory flow, accompanied by the propagation of the hydrothermal wave from the cold sidewall to the hot one, are much smaller than the time-averaged velocity and temperature fields. The corresponding disturbance cells arise in the centre of the liquid pool initially, and extend to the whole region with the increasing imposed temperature difference. The present study reveals the different characteristics of the oscillatory themocapillary flow in the rectangular liquid pool as compared to the cases in other configurations.
\end{abstract}

hydrothermal wave, bifurcation, numerical simulation, microgravity

Citation: Hu W R, Tang Z M, Li K, et al. Numerical study on onset of oscillatory thermocapillary flow in rectangular liquid pool. Sci China Tech Sci, 2010, 53: 1069-1076, doi: 10.1007/s11431-010-0044-1

\section{Introduction}

With an imposed temperature gradient on the free surface, the surface tension gradient of the liquid layer drives the liquid in the vicinity of the free surface from the hot point to the cold point and results in the thermocapillary flow. Thermocapillary flow is the principal natural convection in microgravity and in small scaled configurations on the earth, especially in microgravity the details of the flow can be investigated in depth [1]. There are intensive studies on the thermocapillary flow in a floating zone [2], and theoretical analyses and space experimental results are also available on the thermocapillary flow in a cylindrical container with free surface [3]. On the other hand, studies on the thermocapillary flow in a rectangular liquid pool are limited to terrestrial ones, and successful space experimental studies

*Corresponding author (email: wrhu@imech.ac.cn) are still lacking $[4,5]$. Onset of the oscillatory thermocapillary flow and the corresponding bifurcation process, i.e., the flow transfers from the stationary laminar flow to the oscillatory flow with the increasing imposed temperature difference, is an important subject and of interest in the fluid dynamics, which is closely related to the materials sciences and manufacture.

Theoretical studies on the thermocapillary flow in a rectangular liquid pool were originated by Carpenter and Homsy [6]. They conducted linear stability analysis to obtain the critical temperature difference and coined the concept of the hydrothermal wave which is generally adopted in the explanation of the mechanism of onset of oscillatory thermocapillary flow. Thereafter, intensive studies have focused on the characteristics of the hydrothermal wave [7-9]. Onset of the oscillatory thermocapillary flow in a floating zone or in a cylindrical container is a nonlinear process, i.e., the velocity disturbance of the oscillatory flow is of the same order of the time-averaged velocity while the 
temperature disturbance is smaller, however, still experimentally measurable. On the other hand, the numerical study [10] on the thermocapillary flow in a rectangular liquid pool of $10 \mathrm{cSt}$ silicon oil in microgravity predicted that the maximum ratios of the fluctuation of velocity, temperature and pressure fields to their time-averaged parts are $0.51 \%, 0.05 \%$ and $0.38 \%$ respectively, which are quite small. Moreover, there is a time sequence between the occurrence of the fluctuation and its full development.

In this study, onset of the oscillatory thermocapillary flow and its development in a rectangular liquid pool in microgravity and its relationship to the hydrothermal wave are investigated using the same numerical algorithm as in ref. [10]. The next section is the physical model and mathematical formulation. Section 3 is the numerical results. The conclusions and discussion are provided in the last section.

\section{Physical model and mathematical formula- tion}

As shown in Figure 1, a two dimensional rectangular liquid pool of $10 \mathrm{cSt}$ silicon oil $(\mathrm{Pr}=105.6)$ measures $h=4 \mathrm{~mm}$ and $l=52 \mathrm{~mm}$. The temperature $T_{\mathrm{h}}$ at the left sidewall is higher than $T_{\mathrm{c}}$ at the right sidewall with $\Delta T=T_{\mathrm{h}}-T_{\mathrm{c}}$. The liquid pool has a rigid solid at the bottom and a deformable free surface on the top contacted with a passive gas, which are both assumed to be adiabatic. The liquid is assumed to be incompressible with constant viscosity and thermal diffusivity. The gravity level is zero and the Boussinesq approximation is adopted. The surface tension $\sigma$ on the free surface is a linear function of the temperature $T$, and $-\partial \sigma /$ $\partial T$ is assumed to be constant. $h, \Delta T$ and velocity $U_{\mathrm{r}}=$ $|\partial \sigma / \partial T| \Delta T / \mu$ are adopted as the scales for the length, temperature and velocity, respectively. The pressure is scaled by $\mu U_{\mathrm{r}} / h$, where $\mu$ is the dynamic viscosity.

The non-dimensional vorticity $\omega=\left(\omega_{\xi}, \omega_{\theta}, \omega_{\varsigma}\right)$ and stream-function $\psi=\left(\psi_{\xi}, \psi_{\theta}, \psi_{\varsigma}\right)$ are introduced as

$$
\begin{gathered}
\nabla \times \psi=V, \\
\nabla \times \nabla \times \psi=\omega .
\end{gathered}
$$

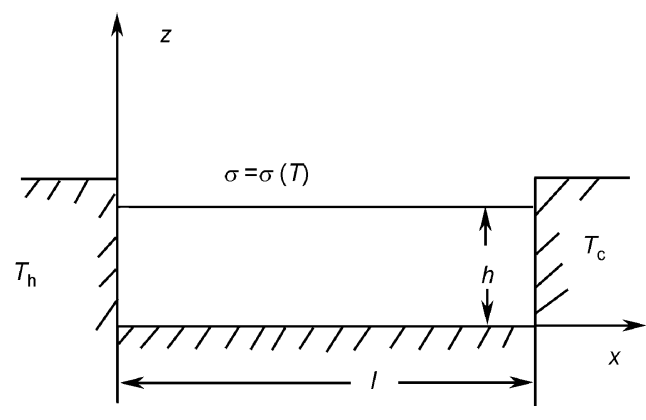

Figure 1 Schematic of a rectangular liquid pool.
The non-dimensional equations and boundary conditions can be expressed by using vorticity $\omega$ and stream-function $\psi$. The continuity equation is satisfied automatically. The momentum equation and thermal equation can be expressed respectively as

$$
\begin{gathered}
\frac{\partial \omega}{\partial t}+V \cdot \nabla \omega-\omega \cdot \nabla V=\frac{1}{R e^{*}} \nabla^{2} \omega, \\
\frac{\partial \Theta}{\partial t}+V \cdot \nabla \Theta=\frac{1}{M a^{*}} \nabla^{2} \Theta .
\end{gathered}
$$

For the two-dimensional problem, the boundary conditions are as follows:

$$
\begin{gathered}
\xi=0: \psi_{\theta}=0, \omega_{\theta}=-\frac{\partial w}{\partial \xi}, \Theta(0, \tau)=f(\tau), \\
\xi=1: \psi_{\theta}=0, \omega_{\theta}=-\frac{\partial w}{\partial \xi}, \Theta(1)=0, \\
\varsigma=0: \psi_{\theta}=0, \omega_{\theta}=\frac{\partial u}{\partial \varsigma}, \frac{\partial \Theta}{\partial n}=0, \\
\varsigma=R(\xi, \tau): \psi=0 .
\end{gathered}
$$

The vorticity on the free surface is determined from the equilibrium of tangential stress:

$$
\begin{aligned}
& \omega=\frac{\left(1+R^{\prime 2}\right)}{\left(1-R^{\prime 2}\right)} \frac{\partial T}{\partial S}+\frac{2 R^{\prime}}{\left(1-R^{\prime 2}\right)} \\
& {\left[\frac{\partial}{\partial \xi}\left(-\frac{\partial \psi}{\partial \zeta}\right)-\frac{\partial}{\partial \zeta}\left(\frac{1}{\xi} \frac{\partial \xi \psi}{\partial \xi}\right)\right]+2 \frac{\partial}{\partial \zeta}\left(-\frac{\partial \psi}{\partial \zeta}\right) .}
\end{aligned}
$$

The temperature boundary condition on the free surface is as follows:

$$
\frac{\partial \Theta}{\partial n}=0
$$

The equilibrium of normal stress is as follows:

$$
\begin{aligned}
& \left(P-P_{\infty}\right)=-\frac{R^{\prime \prime}}{C a\left(1+R^{\prime 2}\right)^{3 / 2}} \\
& +\frac{2}{\left(1+R^{\prime 2}\right)}\left[R^{\prime 2} \frac{\partial U}{\partial \xi}-R^{\prime}\left(\frac{\partial W}{\partial \xi}+\frac{\partial U}{\partial \varsigma}\right)+\frac{\partial W}{\partial \varsigma}\right],
\end{aligned}
$$

where $U$ and $W$ are respectively the non-dimensional velocities in the directions $\xi$ and $\zeta$, where $\xi=x / h$ and $\zeta=z / h$. The free surface is described as $\zeta=R(\xi, \tau)$. The free surface at both the left and right boundaries is located at $\zeta=1$, which means that the method of controlling the height of the liquid layer at the solid/liquid interface is adopted. Here, $n$ is the unit vector in the normal direction of the free surface.

Eqs. (3) and (4) with the initial and boundary conditions are solved by using the hybrid finite element method of fractional steps. The numbers of the cells are 121 and 21 in the directions $x$ and $z$, respectively, and the computation domain is divided into 4800 triangular elements with 2541 nodes. A initial rest plane free surface is assumed and the temperatures at two vertical isothermal sidewalls are kept at the room temperature $T_{0}$ at $t=0$. The temperature at the left 
sidewall is increased from $T_{0}$ to an expected temperature, for example $T_{0}+25^{\circ} \mathrm{C}$, with a heating rate of $0.5^{\circ} \mathrm{C} / \mathrm{s}$, and then is kept constant. The temperature at the right sidewall is kept at $T_{0}$. With the temperature difference, the flow and temperature fields in the liquid pool can be computed. Due to the large Prandtl number of the $10 \mathrm{cSt}$ silicon oil, the free-surface deformation is tiny [5], and then plane free surface is adopted in the present study. Details of the code validation were described in ref. [10] and are ignored here.

\section{Numerical results}

Due to the small fluctuation of the thermocapillary flow in the rectangular pool, it is difficult to observe the fluctuation changes of the velocity and temperature. Similar to ref. [10], the fluctuation in the present study is defined as the difference between the variable and its time-averaged value, e.g.,

$$
u(x, z, t)=u_{0}(x, z)+u^{\prime}(x, z, t),
$$

where $u(x, z, t)$ is the velocity in the $x$ direction, $u_{0}(x, z)$ the corresponding time-averaged velocity and $u^{\prime}(x, z, t)$ the velocity fluctuation where
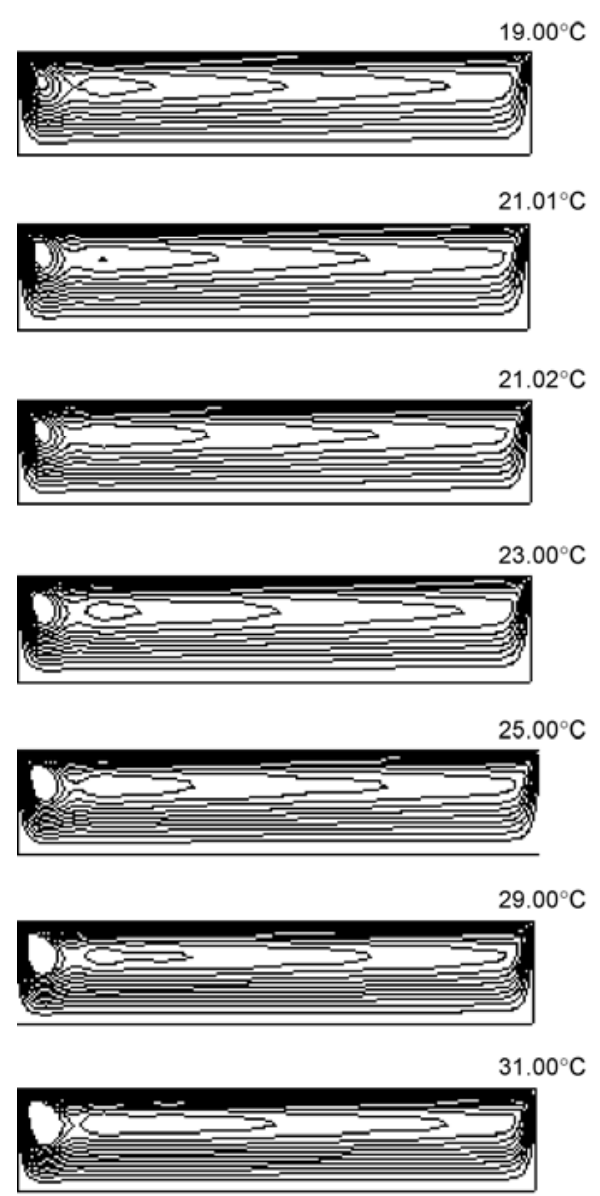

(a)

$$
u_{0}(x, z)=\frac{1}{\tau} \int_{0}^{\tau} u(x, z, t) \mathrm{d} t,
$$

where $\tau$ is the time length much larger than the oscillation period. The numerical results have predicted that the fluctuation of the thermocapillary flow in the rectangular liquid pool is at least two orders less than the corresponding time-averaged value. Figure 2 shows the streamlines $\psi$ (left) and the corresponding fluctuations $\psi^{\prime}$ (right) when the imposed temperature difference goes beyond $19^{\circ} \mathrm{C}$. The stream-function is positive for the solid lines in the plots while negative for the dashed lines. The general trend of the stream-function distribution is similar, i.e., the temperature gradient on the free surface of the liquid pool drives the fluid from the hot sidewall to the cold sidewall while the liquid at the bottom of the liquid pool flows from the cold sidewall to the hot sidewall. When the imposed temperature difference is $\Delta T=21.02^{\circ} \mathrm{C}$, six cells of the stream-function disturbance arise and occupy the most central region of the liquid pool. With the increasing imposed temperature difference, the cells shift to the hot sidewall of the liquid pool, and this is consistent with the propagation direction of the hydrothermal wave. When the imposed temperature differ-
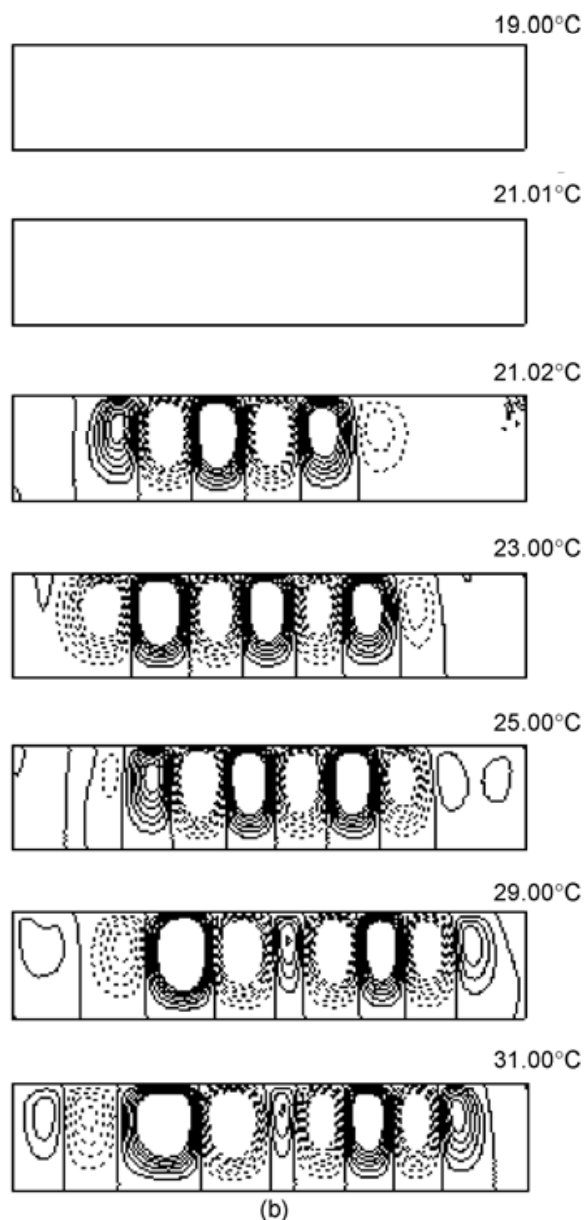

(b)

Figure 2 Evolution of the streamlines $\psi$ (a) and the corresponding fluctuations $\psi^{\prime}$ (b). 
ence is increased to $\Delta T=23.00^{\circ} \mathrm{C}$, seven disturbance cells exist in the liquid pool. When the imposed temperature difference is further increased to $\Delta T=29.00^{\circ} \mathrm{C}, 9$ disturbance cells exist and nearly occupy the whole liquid pool. Note that the stream-function in this process keeps a uni-cellular structure, which is different from the multi-cellular structure for the bifurcation of the Benard convection and the thermcapillary flow in floating half zone with large temperature difference. Figure 3 shows the evolution of the isothermals and the corresponding fluctuations with the increasing imposed temperature difference. Similar to the streamlines, the temperature disturbance cells arise in the center of the liquid pool at $\Delta T=21.02^{\circ} \mathrm{C}$, and progressively occupy the whole liquid pool with the increasing temperature difference. Figure 4 shows the corresponding evolution of the hydrothermal wave. It can be seen that the hydrothermal wave arises at $\Delta T=21.02^{\circ} \mathrm{C}$ propagating from the cold sidewall to the hot sidewall. Note that it is hard to observe the propagation of the traveling wave from the evolutions of the velocity and temperature while the evolutions of the fluctuations, $\psi^{\prime}$ and $T$, clearly exhibit the characteristics of the traveling wave.
Moreover, the propagation region of the traveling wave is approximately equivalent to the extension of the corresponding disturbance cells. Figures 5 and 6 show the evolutions of the velocity and temperature at $x_{\mathrm{c}}=26 \mathrm{~mm}$, the middle point of the free surface, and the corresponding spectra. Such a position selection is to avoid the effect of the sidewalls. It can be seen that the main frequency of the oscillatory velocity for each imposed temperature difference is in consistence with the corresponding oscillatory temperature. Therefore, the onset of oscillatory thermocapillary flow in the rectangular pool is in correspondence with the moment the non-zero fluctuation arisen, and the corresponding imposed temperature difference is the critical temperature difference. In the present study, the critical temperature difference is $\Delta T_{\mathrm{c}}=21.02^{\circ} \mathrm{C}$ with the corresponding critical Marangoni number $M a_{\mathrm{c}}=5695$. It is noted that at the onset of oscillatory thermocapillary flow, the disturbance cells only arise in partial region of the liquid pool. With the increasing imposed temperature difference, the cell number and extension of the disturbances will develop till the whole liquid pool is occupied.
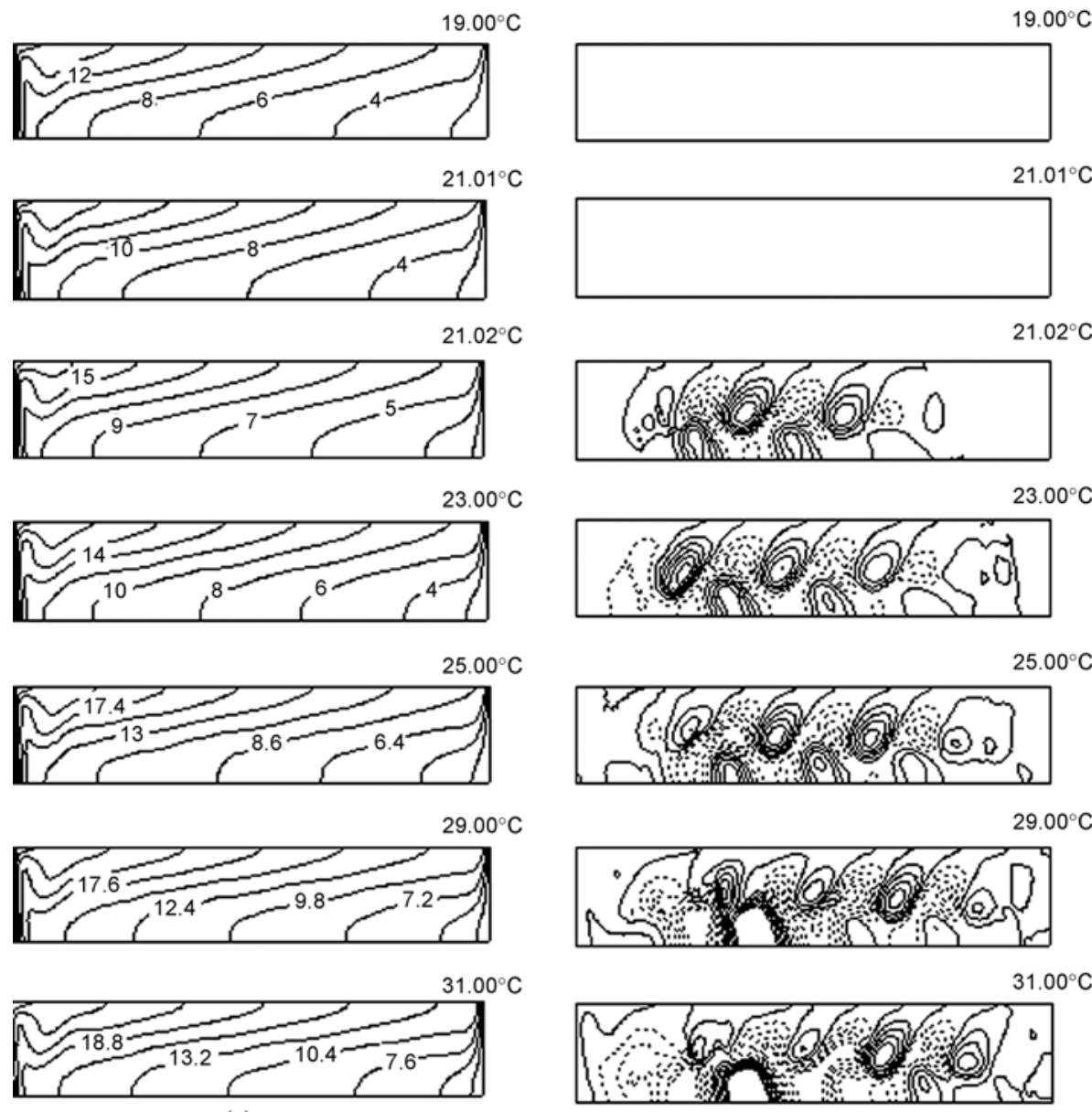

(b)

Figure 3 Evolution of the isothermals (a) and the corresponding fluctuations (b). 

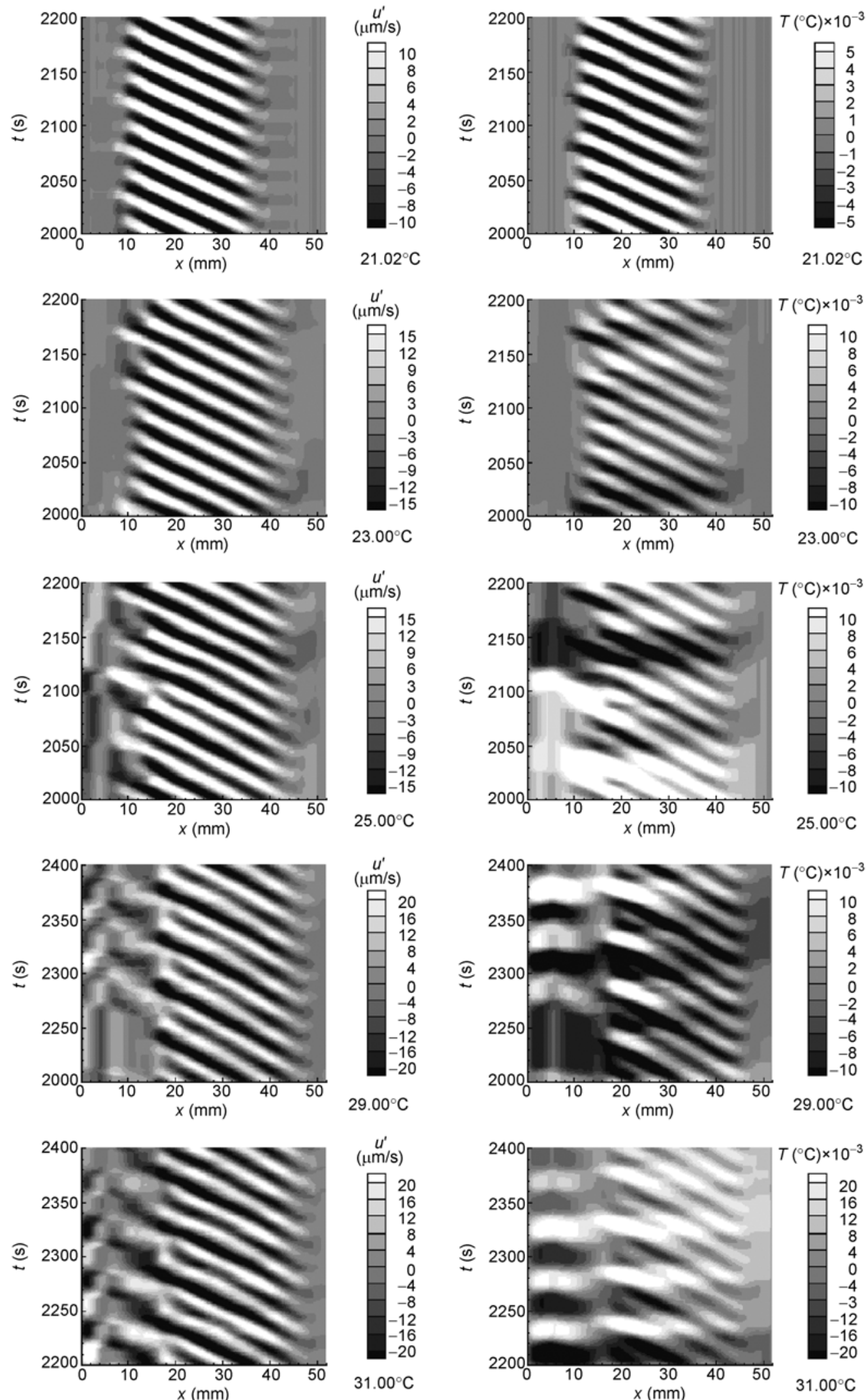

(a)

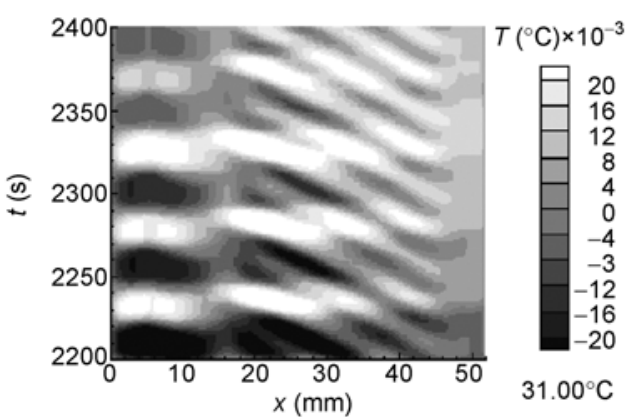

(b)

Figure 4 Hydrothermal waves on the free surface of the liquid pool. (a) Column is the evolution of the velocity disturbance; (b) column is for the temperature disturbance. 

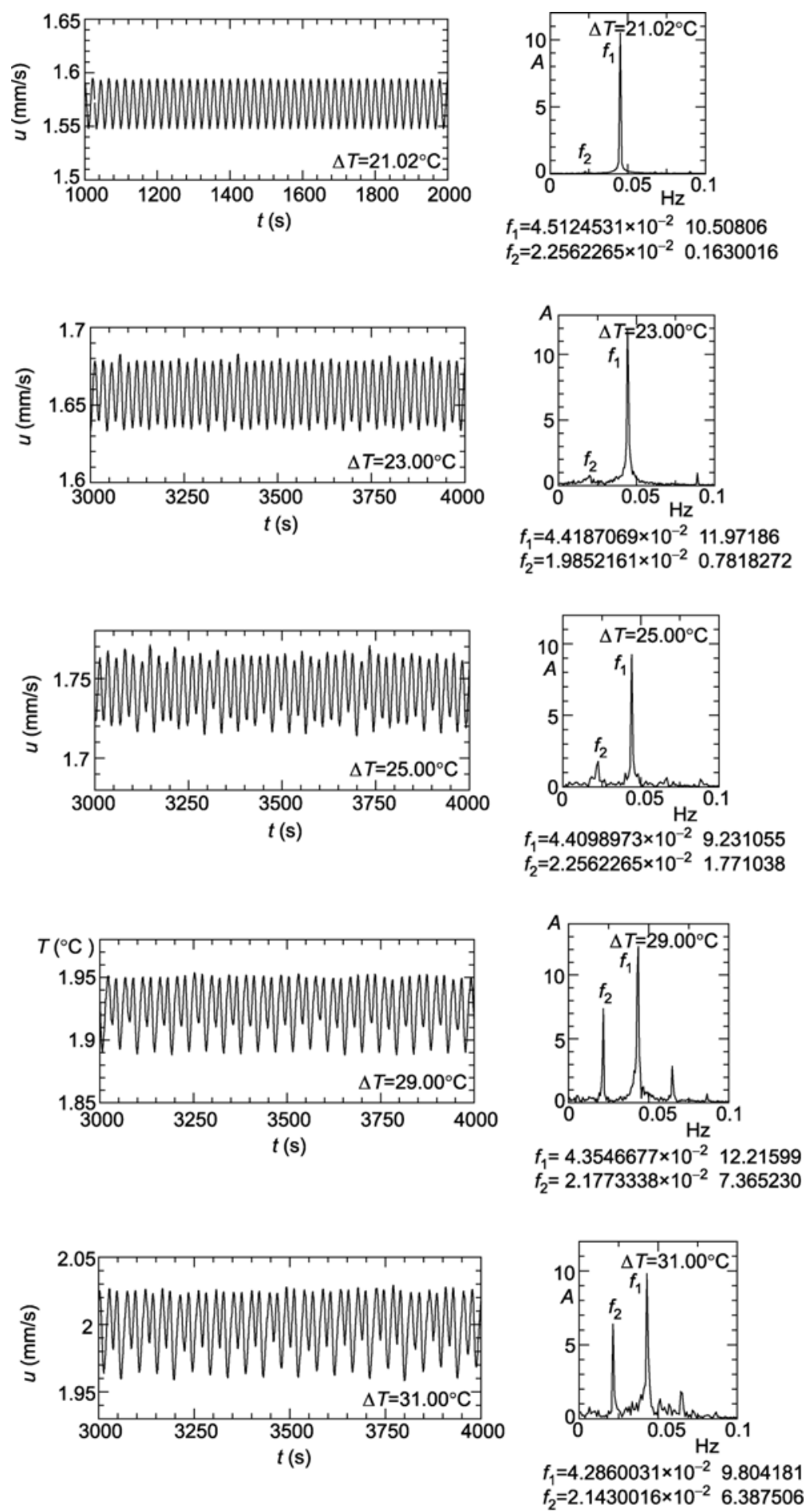

(a)

(b)

Figure 5 Velocity evolution at the middle point of the free surface $\left(x_{\mathrm{c}}=26 \mathrm{~mm}\right)$ (a) and the corresponding spectra (b).

\section{Conclusions and discussion}

Smith and Davis [5] conducted the linear stability analysis on the instability of thermocapillary flow in an infinite liquid layer and promoted the hydrothermal wave instability as the mechanism of the onset of the oscillatory flow. The theory has since attracted extensive attentions, especially in the investigations on the oscillatory themrocapillary flow in a floating half zone. On the other hand, many experimental studies have revealed that the oscillatory themrocapillary flow in the floating half zone is a strongly non-linear process with the velocity fluctuation being the same order of the time-averaged velocity. Generally, the linear stability analysis is inappropriate to be conducted on such a strongly 

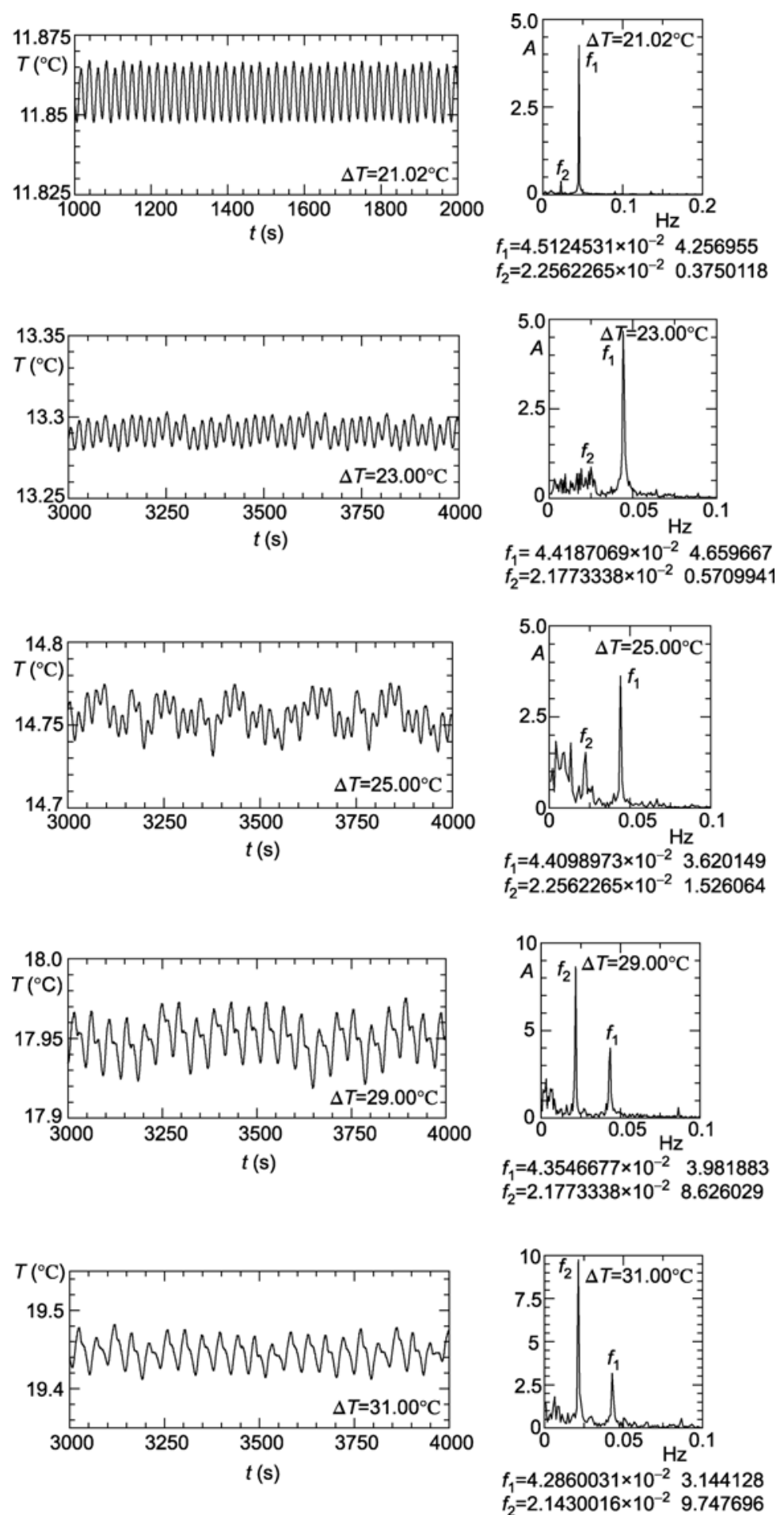

(a)

(b)

Figure 6 Temperature evolution at the middle point of the free surface $\left(x_{\mathrm{c}}=26 \mathrm{~mm}\right)$ (a) and the corresponding spectra (b).

non-linear process. Therefore, 3D direct numerical simulations were conducted on oscillatory themrocapillary flow in the floating half zone, and several mechanisms were promoted [2].

In the present study, onset of the oscillatory thermocapillary flow in a $2 \mathrm{D}$ rectangular liquid pool was numeri- cally studied. Similar to the study on the thermocapillary flow in an infinite liquid layer by Smith and Davis [5], the onset of oscillatory flow was accompanied by the occurrence of the fluctuations of velocity and temperature and the propagation of the hydrothermal wave. In our case, the disturbance cells, however, initially arose in the center of the 
liquid pool and extended to the whole region with the increasing imposed temperature difference. Moreover, the effect of the boundary layers at the sidewall should be taken into account. It is also noted that the characteristics of the themocapillary flow is mainly determined by the timeaveraged velocity and temperature fields while the corresponding fluctuations are very small. This is quite different from the cases in other configurations.

This work was supported by the Knowledge Innovation Project of the Chinese Academy of Sciences (Grant No. KJCX2-YW-L08) and the National Natural Science Foundation of China (Grant No. 1087220250672106).

$1 \mathrm{Hu}$ W R, Xu S C. Fluid Dynamics in Microgravity (in Chinese). Beijing: Science Press, 1999

$2 \mathrm{Hu}$ W R, Tang Z M, Li K. Thermocapillary convection in floating zone. Appl Mech Rev, 2008, 61: 010803

3 Kamotani Y, Ostrach S. Oscillatory thermocapillary flows in open cylindrical containers induced by $\mathrm{Co}_{2}$ laser heating. Int $\mathrm{J}$ Heat \& Mass Transf, 1999, 42: 555-564

4 Schatz M F, Neitzel G P. Experiments on thermocapillary instability. Ann Rev Fluid Mech, 2001, 33: 93-127

5 Smith M K, Davis S H. Instability of dynamic thermocapillary liquid layer, Part 1. Convective instability. J Fluid Mech, 1983, 132: 119-144

6 Carpenter B M, Homsy G M. High Marangoni number convection in a square cavity part II. Phys Fluids A, 1990, 2: 137-149

7 Villers D, Platten J K. Coupled buoyancy and Marangoni convection in acetone: experiments and comparison with numerical simulation. $\mathrm{J}$ Fluid Mech, 1992, 234: 487-510

8 Burguete J, Mukolobwiez N, Daviaud F, et al. Buoyant-thermo capillary instabilities in extended liquid layers subjected to a horizontal temperature gradient. Phys Fluid, 2001, 13: 2773-2787

9 Shevtsova V M, Nepomnyashchy A A, Legros J C. Thermocapillary-buoyancy convection in a shallow cavity heated from the side. Phys Rev E, 2003, 67: 066308

$10 \mathrm{Hu}$ W R, Tang Z M. Hydrothermal wave of large-Prandtl-number fluid in a shallow cavity. Sci China Ser G-Phys Mech Astron, 2007, 50: $1-10$ 